\title{
Emisión de gases de efecto invernadero en la planificación de la generación de energía del Sistema Eléctrico Interconectado del Perú
}

\author{
Douglas Sarango $^{1 *}$, Teresa Velásquez ${ }^{1,2}$, Jorge Gástelo $^{1}$, Guzmán Zorrilla $^{1}$, y Ricardo Alejos ${ }^{3}$ \\ ${ }^{1}$ Universidad Nacional Mayor de San Marcos, Lima, Perú \\ ${ }^{2}$ Universidad Nacional Agraria La Molina, Lima, Perú \\ ${ }^{3}$ Osinergmin-Grt, Lima, Perú
}

Recibido 02 junio 2020 - Aceptado 08 diciembre 2020

\begin{abstract}
Resumen
En esta investigación se determinó el impacto ambiental por uso de combustibles derivados de petróleo, carbón y gas natural en la planificación de la generación de energía del Sistema Eléctrico Interconectado Nacional (SEIN) en el período 2016-2019. Se ha cuantificado la cantidad de $\mathrm{C}, \mathrm{CH}_{4}, \mathrm{~N}_{2} \mathrm{O}, \mathrm{NO}_{\mathrm{x}}, \mathrm{CO}$ y NMCOV, emitidos al medio ambiente por uso de los combustibles Diesel 2, Residual 500, Residual 6, Carbón y Gas Natural. Las emisiones mencionadas están en función del tipo de combustible, la energía anual generada por cada grupo térmico y la aplicación de factores de emisión por quema de combustibles para el sector electricidad obtenidos en 1996 por del Panel Intergubernamental de Cambio Climático (IPCC). Se usó el modelo PERSEO para obtener la planificación de la optimización de la expansión de la generación del SEIN en el período 2016-2019. Este modelo fue desarrollado por la Comisión de Tarifas Eléctricas (CTE) el año 2002 y se viene utilizando por el Organismo Supervisor de la Inversión en Energía y Minería (OSINERGMIN).
\end{abstract}

Palabras claves: gas natural, PERSEO, programación dinámica estocástica, centrales térmicas, centrales hidráulicas, hidrología, contaminantes, impacto ambiental.

\section{Emission of Greenhouse Gases in Plant Planning Power Generation of the SEIN of Peru}

\begin{abstract} hydrology, contaminants, environment impact.

El Sistema Eléctrico Interconectado Nacional (SEIN), está conformado por centrales hidráulicas y centrales térmicas, líneas de transmisión, subestaciones eléctricas, sistemas de compensación reactiva y cargas, que transfieren la energía desde los centrales de generación
\end{abstract}

In this investigation, the environmental impact was determined by the use of fuels derived from petroleum, coal and natural gas in the planning of the generation of energy of the National Interconnected Electrical System (SEIN) in the period 2016-2019. The amount of $\mathrm{C}, \mathrm{CH}_{4}, \mathrm{~N}_{2} \mathrm{O}, \mathrm{NO}_{\mathrm{x}}, \mathrm{CO}$ and NMCOV, emitted into the environment by the use of Diesel 2, Residual 500, Residual 6, Coal and Natural Gas fuels has been quantified. These emissions are depended of fuel type, thermoelectric power plant annual energy generation and other factors of emissions due to burning fuels in the Peru`s Electric Sector obtained for Intergovernmental Panel on Climate Change (IPCC) in 1996. The PERSEO model was used to obtain the optimization planning of the SEIN generation expansion in the period 2016-2019 and it is being used by the Supervisory Agency for Energy and Mining Investment (OSINERGMIN).

Keywords:natural gas, PERSEO, stochastic dynamic programming, thermoelectric power, hydropower,

\section{Introducción}

hidráulica y térmica hacia los centros de demanda (Minen, 2009) y (Sarango, 2009). El SEIN cubre la demanda máxima de energía de nuestro país, que fue de 6275 MW en el año 2015, donde la generación hidráulica y térmica cubren la demanda eléctrica de la zona costera, desde Tacna por el sur hasta el departamento de Tumbes por el norte, y desde la ciudad de Ayacucho hasta la ciudad de Tingo María en la zona central del país, y por la parte sur 
desde Quillabamba en el Cuzco hasta Ilave en Puno (Osinergmin, 2012) y (Sarango 2009).

La energía eléctrica generada por las centrales térmicas es obtenida mediante el uso de gas natural y combustibles derivados de petróleo, como Petróleo Diesel-2, Petróleo Residual 500, Petróleo Residual 6 y Carbón, que son combustibles altamente contaminantes por la emisión como carbono $(\mathrm{C})$, metano $\left(\mathrm{CH}_{4}\right)$, dióxido de nitrógeno $\left(\mathrm{N}_{2} \mathrm{O}\right)$, óxidos de nitrógeno $\left(\mathrm{NO}_{\mathrm{x}}\right)$, monóxido de carbono (CO) y componentes orgánicos volátiles distintos del metano (NMVOC) que son expulsas al aire [Sar09]. Por lo mencionado anteriormente, se planteó la presente investigación para cuantificar los contaminantes emitidos al medio ambiente por su uso de gas natural, derivados de petróleo y carbón en la generación de energía eléctrica en el SEIN.

Para alcanzar este objetivo, se ha realizado el Planeamiento de la Optimización de la Expansión de la Generación del Sistema Interconectado Nacional (SEIN), optimizando los recursos hidráulicos y térmicos existentes y futuros de nuestro país, para cubrir la demanda eléctrica en el período 2016-2019, mediante el uso del modelo PERSEO, que fue desarrollado por la Comisión de Tarifas Eléctricas (CTE) el año 2002. El PERSEO está diseñado para determinar el plan óptimo de la expansión de la generación de mínimo costo de un sistema hidrotérmico, el cual es una secuencia de centrales hidráulicas y térmicas adicionadas al sistema actualmente existente, que es evaluado para obtener el costo de una función objetivo mediante el uso de programación dinámica dualestocástica, y obtener la generación de energía por año de cada central térmica e hidráulica que forma parte del plan de expansión de mínimo costo.

De esta formal los diferentes tipos de centrales térmicas que forman parte del equipamiento de mínimo costo, y los combustibles que utilizan tendrán un impacto en la polución atmosférica, representada por los contaminantes emitidos a la atmósfera para cuya cuantificación es necesario conocer la generación de energía anual (GWh) de cada central térmica dentro del período de planeamiento (2016-2019).

\section{Planteamiento de hipótesis}

a) El uso del gas natural, Petróleo Diesel-2, Petróleo Residual 500, Petróleo Residual 6 y Carbón en la generación de electricidad en el SEIN, período 2016-2019, emite contaminantes al medio ambiente como $\mathrm{C}, \mathrm{CH} 4$, $\mathrm{N}_{2} \mathrm{O}, \mathrm{NOx}, \mathrm{CO}$ y NMVOC.

b) Para determinar las emisiones en el período 2016-2019, se debe obtener el Plan Optimo de Expansión de la Generación de Mínimo Costo del SEIN con el modelo PERSEO, y así obtener la generación de energía anual de cada central térmica y aplicar factores de emisión por quema de combustibles del IPCC y del Inventario Nacional de Gases de Efecto Invernadero.

\section{Materiales y métodos}

\section{Modelo PERSEO}

El SEIN, es un sistema hidrotérmico, los suministradores de energía eléctrica son las centrales hidroeléctricas y termoeléctricas. El consumidor se representa por la demanda total del mercado eléctrico. Las centrales hidroeléctricas turbinan el agua regulada por uno o más embalses, que permite almacenar energía para usarla en diferentes periodos (Osinergmin, 2016) y (Sarango, 2009).

Las características más importantes en la operación de los sistemas hidrotérmicos es el manejo de la energía hídrica almacenada en los embalses del sistema, evitando así los gastos de combustible que ocasionaría la generación térmica. En otras palabras, si se utilizan las reservas del agua para evitar costos por generación térmica en la actualidad, y en el futuro se presenten caudales afluentes bajos, es decir, ocurriera una sequía, podría ocurrir costos por razonamiento muy elevados.

Las ecuaciones de conservación del agua dentro del modelo PERSEO, representan el balance hídrico en cada punto de las cuencas hidrográficas, es así como el volumen final del embalse en la etapa $t$ es igual al volumen inicial (final de la etapa anterior), más las entradas de agua (caudales incrementales afluentes y caudales provenientes de las plantas aguas arriba), menos las perdidas por evaporación, y los volúmenes turbinados y vertidos en el propio embalse (Osinergmin, 2008).

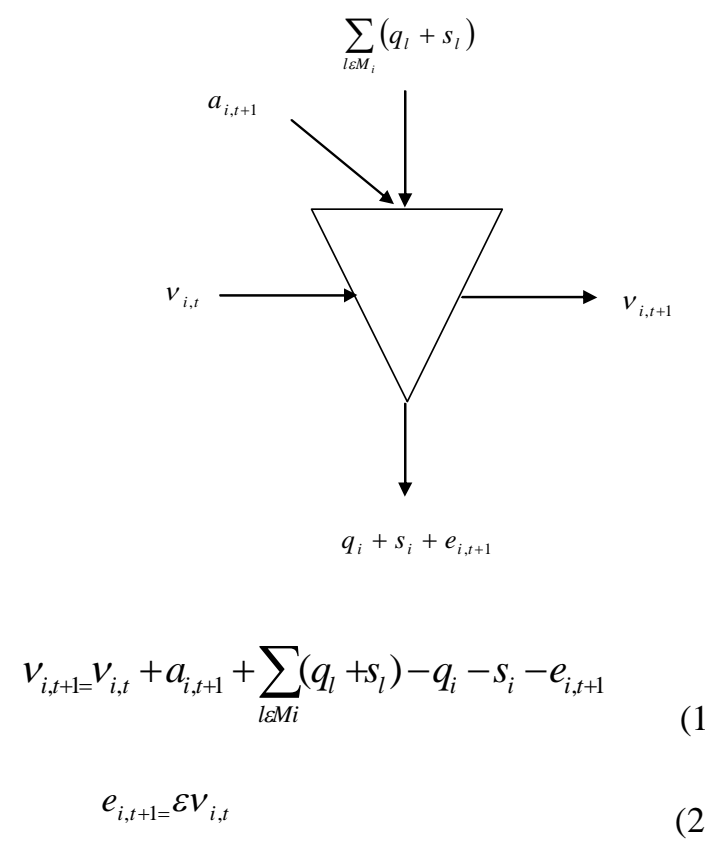


Dónde:

$v_{i, t} \quad$ Volumen de agua almacenado en el embalse de la $i$ ésima planta al inicio de la etapa $t$ (igual al volumen almacenado en la etapa $(t-1)$

$a_{i, t} \quad$ Volumen de agua afluente al embalse de la i-ésima planta durante la etapa $t$

$S_{i, t} \quad$ Volumen de agua vertido por la $i$-ésima planta durante la etapa $t$

$M_{i}$ Conjunto de plantas aguas arriba de la i-ésima planta

$e_{i, t} \quad$ Volumen de agua evaporada en el embalse de la $i$ ésima planta en la etapa $t$

$\varepsilon \quad$ Porcentaje de evaporación respecto del volumen almacenado en el embalse

El modelo matemático de operación de sistemas hidrotérmicos para una secuencia hidrológica $s$, se puede representar a través del modelo recursivo en programación dinámica estocástica, como se indica en la siguiente ecuación (Osinergmin, 2008) y (Sarango, 2009).

$\alpha_{t}\left(v_{t}, A_{t-1}\right)=\mathrm{E}_{A t / A t+1}\left\{\operatorname{Min}\left[\sum_{j=1}^{N j} c o_{j} g t_{j, t}+\alpha_{t+1}\left(v_{t+1}, A_{t}\right)\right]\right\}$

$\sum_{j \varepsilon k} g t_{j, t}+\sum_{i \varepsilon k} \rho_{i} q_{i, j}+\sum_{m \Omega(k)}\left(\left(1-0.5 \gamma_{m k, t}\right) f_{m k, t}-\left(1+0.5 \gamma_{m k, t}\right) f_{m k, t}\right)=d_{k, t}$

$g t_{j, t} \leq \overline{g t}_{j, t}$

$f_{m k, t} \leq \bar{f}_{m k}$

$v_{i, t+1}-v_{i, t}-\sum_{l \varepsilon M_{i}}\left(q_{l}+s_{l}\right)+q_{i}+s_{i}=a_{i, t}-e_{i, t}$

$v_{i, t, k-1}-v_{i, t, k}-\sum_{l \varepsilon M_{i}}\left(q_{l}+s_{l}\right)+q_{i}+s_{i}=a_{i, t}$

$v_{i, t} \leq \bar{v}_{i, t}$

$v_{i, t} \geq \underline{v}_{i, t}$

$v_{i, t, k} \leq \bar{v}_{i}$

$q_{i, t} \leq \bar{q}_{i, t}$

$q_{i, t} \geq \underline{q}_{i, t}$

$r_{i, t} \leq \bar{r}_{i, t}$

$r_{i, t} \geq \underline{r}_{i, t}$

$f_{m k, t}=f_{m k}\left(\theta_{m}, \theta_{k}\right) \geq 0$

$\forall k=1, \ldots \ldots, N_{k} ; i=1, \ldots ., N_{I} ; j=1, \ldots ., N_{j} ; t=1, \ldots ., N_{t}$
Dónde:

i Índice de plantas de generación hidroeléctrica

j Índice de plantas de generación térmica

$\mathrm{k} \quad$ Índice de las barras equivalentes

$\mathrm{s} \quad$ Índice de secuencias hidrológicas

$\mathrm{t} \quad$ Índice de etapas

$N_{k} \quad$ Número total de barras equivalentes en el sistema

$\Omega_{k}$ Conjunto de líneas de transmisión conectadas a la barra equivalente $k$

$f_{m k, t}$ Flujo de energía de la barra equivalente $m$ para la barra equivalente $k$ en la etapa $t$

$\gamma_{m k, t}$ Coeficiente de pérdidas de flujo de transmisión de $m$ para $k$ durante la etapa $t$, expresado en $\%$ del flujo actual

$d_{k, t}$ Demanda de energía en la barra equivalente $k$ durante la etapa $t$

$\bar{v}_{i, t} \quad$ Volumen del embalse mínimo

$\underline{v}_{i, t} \quad$ Volumen del embalse máximo

$\bar{v}_{i} \quad$ Volumen máximo en los reservorios diarios

$q_{i, t}$ Volumen turbinado en la i-ésima planta hidroeléctrica durante la etapa $t$

$\bar{q}_{i, t} \quad$ Volumen turbinado máximo

$\underline{q}_{i, t} \quad$ Volumen turbinado mínimo

$r_{i, t} \quad$ Caudales regulados

$\underline{r}_{i, t}$ Caudales regulados mínimos de irrigación, navegación o recreación

$\bar{r}_{i, t}$ Caudales regulados máximos caso de grandes avenidas

\section{Información utilizada}

\section{Demanda eléctrica}

Se consideran los resultados de proyección de la demanda eléctrica de estudio de la Fijación Tarifaria de mayo 2017, periodo 2016-2019 (Coes, 2017).

\begin{tabular}{|c|c|c|}
\hline Año & $\begin{array}{c}\text { Máxima } \\
\text { Demanda } \\
\text { Potencia } \\
\text { (MW) }\end{array}$ & $\begin{array}{c}\text { Máxima } \\
\text { Demanda } \\
\text { Energía } \\
\text { (GWh) }\end{array}$ \\
\hline 2016 & 6644.00 & 48369.00 \\
\hline 2017 & 7087.00 & 51693.00 \\
\hline 2018 & 7563.00 & 55329.00 \\
\hline 2019 & 8039.00 & 58778.00 \\
\hline
\end{tabular}

Tabla 1: Proyección de demanda eléctrica del SEIN (2016-2019) 


\section{Situación Actual del Sistema Eléctrico Interconectado Nacional (SEIN)}

La situación actual del SEIN está representada por las características técnicas y económicas de las centrales hidráulicas y térmicas existentes a diciembre de 2016 (Coes, 2017). (Tabla 2 y Tabla 3).

\begin{tabular}{clll}
\hline $\mathbf{N}^{\circ}$ & \multicolumn{1}{c}{ Razón Social } & \multicolumn{1}{c}{ Abreviatura } & \multicolumn{1}{c}{ Estado } \\
\hline 1 & Aguas y Energía Perú S.A. & AYEPSA & Voluntario \\
2 & Cerro del Aguila S.A. & CERRO DEL AGUI Obligatorio \\
3 & Chinango S.A.C & CHINANGO & Obligatorio \\
4 & Cia. Eléctrica El Platanal S.A. & CELEPSA & Obligatorio \\
5 & Duke Energy Egeneor S. en C. por A. & EGENOR & Obligatorio \\
6 & Eléctrica Santa Rosa S.A.C. & SANTA ROSA & Voluntario \\
7 & Elécrica Yanapampa S.A.C. & YANAPAMPA & Voluntario \\
8 & EGE Eléctrica Arequipa S.A. & EGASA & Obligatorio \\
9 & EGE Del Sur S.A. & EGESUR & Obligatorio \\
10 & EGE Machupicchu S.A. & EGEMSA & Obligatorio \\
11 & EGE San Gabán S.A. & SAN GABÁN & Obligatorio \\
12 & EGE Huallaga S.A. & HUALLAGA & Obligatorio \\
13 & EGE Huanza S.A. & EGEHUANZA & Obligatorio \\
14 & EGE Río Roble S.A. & RIO ROBLE & Voluntario \\
15 & EGE Del Perú S.A. & ELECTROPERU & Obligatorio \\
16 & ENEL Generación Perú S.A.A. & ENELPERU & Obligatorio \\
17 & ENGIE Energía Perú & ENGIE & Obligatorio \\
18 & Hidroeléctrica Huanchor S.A.C. & HUANCHOR & Voluntario \\
19 & Hidroeléctrica Santa Cruz S.A.C. & SANTA CRUZ & Voluntario \\
20 & Statkraft Perú S.A. & STATKRAFT & Obligatorio \\
21 & Sindicato Energético S.A. & SINERSA & Voluntario \\
\hline
\end{tabular}

Tabla 2: Grandes empresas generadoras hidráulicas del SEIN al 2016

\begin{tabular}{lcl}
\hline \multicolumn{1}{c}{ Empresa } & $\begin{array}{c}\text { Potencia } \\
\text { Efectiva } \\
\text { (MW) }\end{array}$ & Combustible \\
\hline EEPSA & 155.46 & Gas Natural \\
EGENOR & 55.30 & Diesel B5 \\
EDEGEL & 997.94 & Gas Natural \\
EDEGEL & 217.54 & Diesel B5 \\
SHOUGESA & 64.00 & R 500, D B5 \\
TERMOSELVA & 176.29 & Gas Natural \\
ELECTROPERU & 93.60 & Residual 6, D B5 \\
ENERSUR & 1373.53 & Gas Natural \\
ENERSUR (NES Ilo & 600.00 & Diesel B5 \\
ENERSUR (RF Ilo) & 497.00 & Diesel B6 \\
KALLPA & 1635.93 & Gas Natural \\
FENIX POWER & 947.51 & Gas Natural \\
SAN GABAN & 4.09 & Diesel B5 S-50 \\
EEPSA & 193.40 & Diesel B5 \\
COBRA & 215.00 & Diesel B5 \\
EGASA & 28.85 & Residual 500 \\
SAN GABAN & 1.32 & Diesel B5 S-51 \\
TERMOCHILCA & 501.25 & Gas Natural \\
SDE PIURA & 26.43 & Gas Natural \\
I\&E del Peru & 58.00 & Diesel B5 S-50 \\
SAMAY (Pto Bravo) & 612.85 & Diesel B5 S-50 \\
NUEVA ESPERAN2 & 135.00 & Gas Natural \\
CERRO VERDE & 178.78 & Diesel B5 \\
\hline & &
\end{tabular}

Tabla 3: Principales empresas generadoras térmicas del SEIN al 2016
Oferta de generación hidráulica y térmica comprometida y en proyecto

Para el planeamiento de la expansión de la generación del SEIN, período 2016-2019, la oferta de generación hidráulica y térmica de potencia se muestra en la Tabla 4. Se toma en cuenta proyectos de generación hidráulica igual o menor a 20.0 MW como proyectos RER (Coes, 2017).

\begin{tabular}{ccccc}
\hline \multirow{2}{*}{ Año } & \multicolumn{2}{c}{ Hidro $(\mathbf{M W})$} & Termico & Combustible \\
\cline { 2 - 4 } & $\begin{array}{c}\text { Proyecto } \\
\text { Grande }\end{array}$ & $\begin{array}{c}\text { Proyecto } \\
\text { RER }\end{array}$ & & \\
\hline 2016 & 456.0 & 190.0 & 635.7 & Gas Natural \\
2017 & 34.9 & - & - & - \\
2018 & 140.2 & 257.6 & - & - \\
2019 & - & 23.3 & - & - \\
\hline TOTAL & 631.1 & 470.9 & 635.7 & - \\
\hline
\end{tabular}

Tabla 4: Oferta de potencia de centrales hidroeléctricas y térmicas en proyecto del SEIN (2016-2019)

\section{Metodología}

La metodología seguida para el planeamiento de la expansión de la generación de SEIN es la siguiente:

- Determinación de la demanda eléctrica y su proyección en el período de planeamiento.

- Premisas económicas para el planeamiento de la expansión de la generación del SEIN.

- Análisis de los recursos hídricos de las cuencas hidrográficas pertenecientes al SEIN.

- Determinación de las características técnicas y económicas de las centrales hidráulicas y centrales térmicas existentes del SEIN.

- Determinación de las opciones de generación hidráulica y térmica comprometidas en entrar a operar en el SEIN dentro del período de estudio.

- Determinación de las características técnicas y económicas de las centrales hidráulicas y centrales térmicas en proyecto para la expansión del SEIN.

- Elección del modelo PERSEO para el planeamiento de la expansión de la generación del SEIN.

- Elaboración de los archivos de datos de entrada del modelo PERSEO.

- Obtener la generación de energía de las centrales térmicas para el periodo de planeamiento.

- Determinar la emisión $\mathrm{C}, \mathrm{CH}_{4}, \mathrm{~N}_{2} \mathrm{O}, \mathrm{NO}_{\mathrm{x}}, \mathrm{CO}$ y NMCOV mediante la aplicación de factores de emisión a la energía generada por las centrales térmicas del SEIN en el periodo de estudio.

\section{Resultados y Discusión}

Proyección de la Demanda Eléctrica (2016-2019)

La proyección de la demanda de potencia para el período 2016-2019 es la obtenida en la Fijación Tarifaria mayo 2017, siendo la demanda máxima de potencia de 6644 MW para el 2016, de 7087 MW para el 2017, 7563 
MW para el año 2018 y en el 2019 de 8039 MW (Figura $1)$.

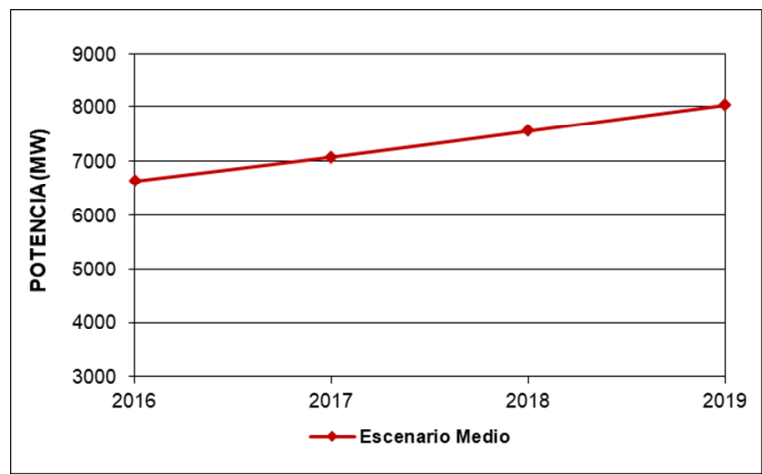

Figura 1: Proyección de demanda de potencia del SEIN, período 2016-2019.

Elaboración de los Archivos de datos de entrada del modelo PERSEO

Con la información de proyección demanda eléctrica para el escenario medio en el período 2016-2019, los datos técnicos y económicos de las centrales hidroeléctricas y térmicas existentes, y los proyectos de generación hidráulica y proyectos de generación térmica del SEIN, las premisas y criterios económicos para el planeamiento de la expansión de la generación eléctrica, se elaboraron los archivos de datos de entrada del modelo PERSEO. De estos archivos se tiene, por ejemplo: SEIN.hid contiene las series de caudales mensuales naturales de las cuencas hidrográficas comprometidas con el SEIN, para el periodo 1965-2016.

Planeamiento de la expansión de la generación del SEIN

El modelo PERSEO, determina la política de optimización del planeamiento de expansión de generación de mínimo costo del SEIN. Esta expansión del sistema es técnicamente factible y garantizar el cubrimiento de la demanda dentro del período de estudio con niveles adecuados de confiabilidad, calidad y seguridad.

Considerando el escenario de demanda media (20162019), se determinó el programa de equipamiento de expansión de mínimo costo del SEIN, (Véase Tabla 5). Los resultados indican las nuevas centrales hidráulicas y térmicas del SEIN para el período 2016-2019, así como su potencia y fecha de entrada en operación (Tabla 5).

\begin{tabular}{|c|c|c|}
\hline $\begin{array}{c}\text { Fecha de } \\
\text { ingreso }\end{array}$ & Proyecto & $\begin{array}{c}\text { Potencia } \\
\text { (MW) }\end{array}$ \\
\hline oct 2016 & C.H. Chaglla & 456.00 \\
\hline $\operatorname{dic} 2016$ & C.H. 8 de Agosto (Huanuco) & 19.00 \\
\hline oct 2016 & C.T. Chilca 1 CS (Amp.) & 35.70 \\
\hline nov 2016 & C.T. Ilo-Nodo Energetico & 600.00 \\
\hline abr 2017 & C.H. Potrero & 19.90 \\
\hline $\operatorname{dic} 2017$ & C.H. Yarucaya (Lima) & 15.00 \\
\hline ene 2018 & C.H. La Virgen (Junin) & 84.00 \\
\hline ene 2018 & C.H. Angel I & 20.00 \\
\hline ene 2018 & C.H. Angel II & 20.00 \\
\hline ene 2018 & C.H. Angel III & 20.00 \\
\hline ene 2018 & C.H. Santa Lorenza & 18.70 \\
\hline feb 2018 & C.H. Renovandes & 20.00 \\
\hline $\operatorname{mar} 2018$ & C.H. Tulumayo IV & 56.20 \\
\hline abr 2018 & C.H. Manta & 19.80 \\
\hline jul 2018 & C.H. Marañon & 18.40 \\
\hline jul 2018 & C.H. Karpa & 19.00 \\
\hline jul 2018 & C.H. Hydrika 5 (Ancash) & 10.00 \\
\hline jul 2018 & C.H. Hydrika 2 (Ancash) & 4.00 \\
\hline ago 2018 & C.H. Huatziroki (Ayacucho) & 11.10 \\
\hline nov 2018 & C.H. Hydrika 3 (Ancash) & 10.00 \\
\hline oct 2018 & C.H. Hydrika 4 (Ancash) & 8.00 \\
\hline nov 2018 & C.H. Carhuac (Huanza- & 20.00 \\
\hline nov 2018 & C.H. Hydrika 1 (Ancash) & 6.60 \\
\hline dic 2018 & C.H. Laguna Azul & 20.00 \\
\hline dic 2018 & C.H. Colca & 12.05 \\
\hline ene 2019 & C.H. Zaña & 13.20 \\
\hline jun 2019 & C.H. Pallca & 10.10 \\
\hline
\end{tabular}

Tabla 5: Programa de equipamiento del SEIN (2016-2019)

Emisiones por uso del gas natural y combustibles derivados de petróleo en la generación de energía del SEIN (2016-2019)

El efecto en el medio ambiente por el uso de gas natural, Petróleo Diesel-2, Petróleo Residual 500, Petróleo Residual 6 y Carbón en las centrales térmicas del SEINpara la generación de energía, está representado por la cantidad de emisiones de $\mathrm{C}, \mathrm{CH}_{4}, \mathrm{~N}_{2} \mathrm{O}, \mathrm{NO}_{\mathrm{x}}, \mathrm{CO}$ y NMCOV por año de cada central térmica existente y en proyecto en el periodo 2016-2019.

La cantidad de emisiones son obtenidas por aplicación de factores de emisión a la generación de energía anual de cada central térmica, estos factores corresponden a la categoría de quema de combustibles del Sub Sector Electricidad obtenidos por del Panel Intergubernamental de Cambio Climático (IPCC) en 1996 y revisados en el 2006 (Tabla 6). 


\begin{tabular}{lcccccc}
\hline & \multicolumn{5}{c}{ Factor de Emisión } \\
\cline { 2 - 7 } \multicolumn{1}{c}{ Combustible } & $\begin{array}{c}\mathbf{C} \\
(\mathbf{t} / \mathbf{T J})\end{array}$ & $\begin{array}{c}\mathbf{C H}_{4} \\
(\mathbf{k g} / \mathbf{T J})\end{array}$ & $\begin{array}{c}\mathbf{N}_{2} \mathbf{O} \\
(\mathbf{k g} / \mathbf{T J})\end{array}$ & $\begin{array}{c}\mathbf{N O x} \\
(\mathbf{k g} / \mathrm{TJ})\end{array}$ & $\begin{array}{c}\mathbf{C O} \\
(\mathbf{t} / \mathbf{T J})\end{array}$ & $\begin{array}{c}\text { NMVOC } \\
(\mathbf{k g} / \mathbf{T J})\end{array}$ \\
\hline Petróleo Diesel 2 & 20.2 & 1.0 & 0.6 & 200.0 & 15.0 & 5.0 \\
Petróleo Residual 50 & 21.1 & 3.0 & 0.6 & 200.0 & 15.0 & 5.0 \\
Petróleo Residual 6 & 21.1 & 3.0 & 0.6 & 200.0 & 15.0 & 5.0 \\
Carbón & 25.8 & 10.0 & 1.4 & 300.0 & 20.0 & 5.0 \\
Gas Natural & 15.3 & 4.0 & 0.1 & 150.0 & 20.0 & 5.0 \\
\hline
\end{tabular}

Tabla 6: Factores de emisión de quema de combustibles en el subsector electricidad - IPCC.

Se presentan los resultados de la generación de energía producida por las centrales térmicas existente y en proyecto en el periodo 2016-2019, obtenida con el modelo PERSEO. Estos muestran para cada año, que la generación térmica usando Petróleo Diesel 2 es menor a la generada con Gas Natural, siendo este último el combustible más usado para generar energía térmica del SEIN, no siendo utilizados el petróleo residual 500, petróleo residual 6 y el carbón (Tabla 7).

\begin{tabular}{lcrrrr}
\hline \multicolumn{1}{c}{ Fuente } & Unidad & $\mathbf{2 0 1 6}$ & $\mathbf{2 0 1 7}$ & $\mathbf{2 0 1 8}$ & $\mathbf{2 0 1 9}$ \\
\hline Petróleo Diesel 2 & GWh/año & 455 & 372 & 410 & 535 \\
Petróleo Residual 500 & GWh/año & 0 & 0 & 0 & 0 \\
Petróleo Residual 6 & GWh/año & 0 & 0 & 0 & 0 \\
Carbón & GWh/año & 0 & 0 & 0 & 8 \\
Gas Natural & GWh/año & 20,138 & 19,765 & 19,773 & 21,352 \\
\hline Total & & 20,592 & 20,137 & 20,183 & 21,895 \\
\hline
\end{tabular}

Tabla 7: Generación de energía térmica en el SEIN (2016-2019)

La cantidad total de emisiones de $\mathrm{C}, \mathrm{CH}_{4}, \mathrm{~N}_{2} \mathrm{O}, \mathrm{NO}_{\mathrm{x}}$, $\mathrm{CO}$ y NMCOV emitidos a la atmósfera por el uso del Gas Natural y Petróleo Diesel 2 en la generación de energía en el SEIN, en el período de estudio, se muestran en la Tabla 8.

\begin{tabular}{|c|c|c|c|c|c|c|c|}
\hline \multirow{2}{*}{$\begin{array}{l}\text { Foco de } \\
\text { Emisión }\end{array}$} & \multirow{2}{*}{$\begin{array}{c}\text { Energia } \\
\text { Generada } \\
\text { (TJ) }\end{array}$} & \multicolumn{6}{|c|}{ Emision $\times 10^{3}$} \\
\hline & & $\begin{array}{c}\mathrm{C} \\
(\mathrm{t})\end{array}$ & $\begin{array}{l}\text { CH4 } \\
\text { (kg) }\end{array}$ & $\begin{array}{l}\mathrm{N} 20 \\
(\mathrm{~kg})\end{array}$ & $\begin{array}{l}\text { NOx } \\
\text { (kg) }\end{array}$ & $\begin{array}{l}\text { CO } \\
(\mathrm{t})\end{array}$ & $\begin{array}{c}\text { NMVOC } \\
(\mathrm{kg})\end{array}$ \\
\hline Petróleo Diesel 2 & 21.17 & 428 & 21 & 13 & 4234 & 318 & 106 \\
\hline Petróleo Residual 500 & 0 & 0 & 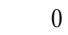 & 0 & 0 & 0 & 0 \\
\hline Petróleo Residual 6 & 0 & 0 & 0 & 0 & 0 & 0 & 0 \\
\hline Carbón & 0 & 0 & 0 & 0 & 0 & 0 & 0 \\
\hline Gas Natural & 291.70 & 4463 & 1167 & 29 & 43755 & 5834 & 1459 \\
\hline
\end{tabular}

Tabla 8: Contaminantes emitidos por uso de Diesel 2 y gas natural en el SEIN (2016-2019)
En la Figura 2, se observa gráficamente estos resultados, siendo los más representativos la emisión de carbono (C) y monóxido de carbono (CO), donde es mayor el uso del gas natural en el SEIN.

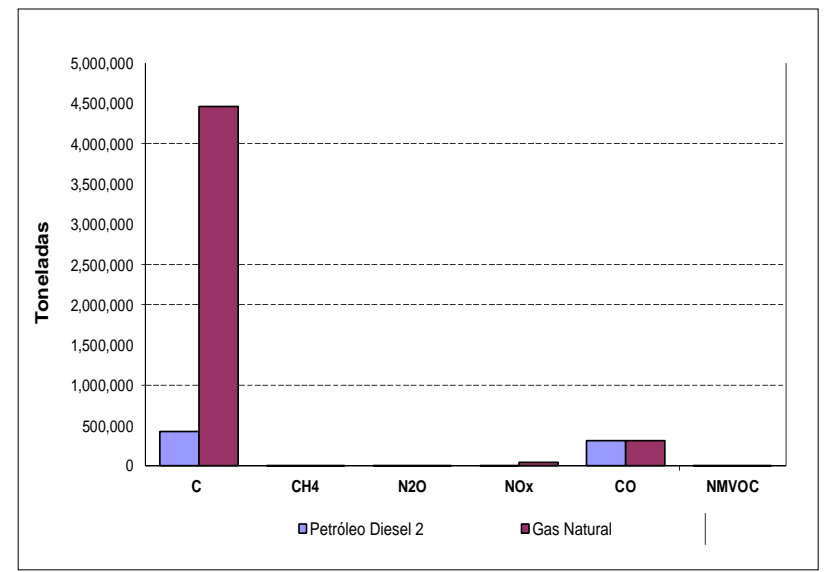

Figura 2: Comparación de contaminantes emitidos por uso de Diesel 2 y gas natural en el SEIN (2016-2019).

\section{Conclusiones}

El uso del gas natural como combustible para la generación de energía eléctrica en el SEIN, no evita la contaminación del medio ambiente, ya que existe emisión de carbono $(\mathrm{C})$, metano $\left(\mathrm{CH}_{4}\right)$, dióxido de nitrógeno $\left(\mathrm{N}_{2} \mathrm{O}\right)$, óxidos de nitrógeno $\left(\mathrm{NO}_{\mathrm{x}}\right)$, monóxido de carbono (CO) y componentes orgánicos volátiles distintos del metano (NMVOC), y que también son generados por uso de combustibles derivados de petróleo, como es el Petróleo Diesel-2.

En el periodo de estudio (2016-2019), no se usan para la generación de energía eléctrica el Petróleo Residual 500, Petróleo Residual 6 y el Carbón.

La emisiones obtenidas son perjudiciales para el medio ambiente y constituyen una parte importante en la generación de gases de efecto invernadero en nuestro país, esto considerando los resultados del Inventario Nacional de Gases de Efecto Invernadero (ENGI) del año 2014 (Minan, 2014), donde se indica que el Sector Energía, es el segundo sector con mayores emisiones de GEI reportadas en el INGEI 2012, con un total de 30\% del total de emisiones, de este porcentaje la quema de combustibles es el $91.22 \%$, y donde la industria de la energía - Generación de Electricidad en el SEIN y Sistemas Aislados contribuyen con el $32.13 \%$ de estas emisiones por quema de combustibles. 


\section{Referencias}

COES-SEIN (2017). Comité de Operación Económica del Sistema Interconectado Nacional. Estudio Técnico Económico de Determinación de Precios de Potencia y Energía en Barras para la Fijación Tarifaría Período Mayo 2018 - Abril 2019. Lima, Perú.

IPCC (1996). Panel Intergubernamental de Cambio Climatico. Directrices para Inventarios Nacionales de Gases de Efecto Invernadero.

IPCC (2006). Panel Intergubernamental de Cambio Climatico. Directrices para Inventarios Nacionales de Gases de Efecto Invernadero para los Sectores Energía y Procesos Industriales.

MINEM (2009). Ministerio de Energía y Minas del Perú. Plan Referencial de Electricidad del Perú 2008-2017. Dirección General de Electricidad, Lima-Perú.

MINEM (1992). Ministerio de Energía y Minas del Perú. Decreto Ley $N^{\circ}$ 25844. Ley de Concesiones Eléctricas y sus Modificatorias. 211 p. Lima, Perú.

MINEM (1993). Ministerio de Energía y Minas del Perú. DecretoSupremo No. 009-93-EM. Reglamento de la Ley de Concesiones Eléctricas y sus Modificatorias. 198 p. Lima, Perú.
MINAM (2014). Ministerio del Ambiente del Perú. Inventario Nacional de Gases de Efecto Invernadero del Año 2014 y actualizaciones de las estimaciones de los años 2000, 2005, 2010 y 2012. Lima, Perú.

OSINERGMIN-GART (2008). Organismo Supervisor de la Inversión en Energía y Minería del Perú. Metodología y Manual de Uso del modelo PERSEO. Lima, Perú.

OSINERGMIN (2012). Organismo Supervisor de la Inversión en Energía y Minería del Perú.PR-41: Procedimiento Técnico del Comité de Operación Económica del SEIN. Información Hidrológica para la Operación del SEIN. Resolución $N^{\circ}$ 153-2012-OS/CD. Lima, Perú.

OSINERGMIN (2016). Organismo Supervisor de la Inversión en Energía y Minería del Perú. Proceso de Fijación Tarifas en Barra delCosto Marginal Promedio de la Energía del Sistema Eléctrico Interconectado Nacional - SEIN, Période mayo 2018 - abril 2019. Lima, Perú

Sarango, J. y Velásquez, B. (2009). Uso de Series Sintéticas de Caudales Mensuales en el Cálculo del Costo marginal de Energía del Sistema Eléctrico Interconectado Nacional del Perú. Revista de Investigación de Física, Vol 12, No. 1, pp 59-69. 